\title{
Weak charge of the proton: loop corrections to parity-violating electron scattering
}

\author{
W. Melnitchouk \\ Jefferson Lab, 12000 Jefferson Avenue, Newport News, Virginia 23606, USA
}

\begin{abstract}
I review the role of two-boson exchange corrections to parity-violating elastic electronproton scattering. Direct calculations of contributions from nucleon and $\Delta$ intermediate states show generally small, $\mathscr{O}(1-2 \%)$, effects over the range of kinematics relevant for strangeness form factor measurements. For the forward angle Qweak experiment at Jefferson Lab, which aims to measure the weak charge of the proton, corrections from the $\gamma Z$ box diagram are computed within a dispersive approach and found to be sizable at the $E \sim 1 \mathrm{GeV}$ energy scale of the experiment.
\end{abstract}

\section{PARITY-VIOLATING ELECTRON SCATTERING}

Parity-violating elastic electron-proton scattering has become a widely-used tool to probe novel aspects of the structure of the nucleon, such as its strangeness content $[1,2]$, its weak charge [3], and even as an indirect test of physics beyond the Standard Model [4]. By polarizing the incident electron and measuring the difference between rightand left-handed electrons scattering from unpolarized protons, the parity-violating (PV) asymmetry can be defined as

$$
A_{\mathrm{PV}}=\frac{\sigma_{R}-\sigma_{L}}{\sigma_{R}+\sigma_{L}}
$$

where $\sigma_{R(L)}$ is the cross section for a right- (left-) hand polarized electron. The purely electromagnetic contribution cancels in the numerator, so that the asymmetry becomes proportional to the parity-violating part of the interference cross section, $2 \Re e\left(\mathscr{M}_{\gamma}^{*} \mathscr{M}_{Z}\right)$, involving the interference of the Born amplitude $\mathscr{M}_{\gamma}$ with the product of vector and axial-vector currents in $\mathscr{M}_{Z}$ (the vector-vector and axial-axial parts of $\mathscr{M}_{Z}$ also cancel in the asymmetry).

More explicitly, the PV asymmetry can be written in terms of the proton electroweak form factors as

$$
A_{\mathrm{PV}}=\left(\frac{G_{F} t}{4 \sqrt{2} \pi \alpha}\right) \frac{g_{A}^{e}\left(\varepsilon G_{E}^{\gamma p} G_{E}^{Z p}+\tau G_{M}^{\gamma p} G_{M}^{Z p}\right)+g_{V}^{e} \varepsilon^{\prime} G_{M}^{\gamma p} G_{A}^{Z p}}{\varepsilon\left(G_{E}^{\gamma p}\right)^{2}+\tau\left(G_{M}^{\gamma p}\right)^{2}}
$$

where $t$ is the four-momentum transfer squared to the proton, $\tau=-t / 4 M^{2}$, with $M$ the proton mass, $\varepsilon^{-1}=1+2(1+\tau) \tan ^{2}(\theta / 2)$ and $\varepsilon^{\prime}=\sqrt{\tau(1+\tau)\left(1-\varepsilon^{2}\right)}$ are kinematical parameters, and $\theta$ is the electron scattering angle. The coupling constants $g_{A}^{e}$ and $g_{V}^{e}$ are the axial and vector couplings of the $Z$ boson to the electron, respectively, $G_{F}$ is the Fermi constant, and $\alpha=e^{2} / 4 \pi$ the electromagnetic fine structure constant. 
For a proton target the weak electric (magnetic) vector form factor $G_{E(M)}^{Z p}$ can be related by isospin symmetry to the electromagnetic form factors of the proton and neutron by

$$
G_{E(M)}^{Z p}=\left(1-4 \sin ^{2} \theta_{W}\right) G_{E(M)}^{\gamma p}-G_{E(M)}^{\gamma n}-G_{E(M)}^{s},
$$

where $G_{E(M)}^{s}$ are the contributions from strange quarks. Measurement of the PV asymmetry $A_{\mathrm{PV}}$ as a function of the scattering angle $\theta$ allows one to extract combinations of the strange form factors, given knowledge of the proton and neutron electromagnetic form factors, which historically was one of the main motivations for the PV experiments. Reliable extractions of the form factors require precise knowledge of the radiative corrections to the PV scattering associated with higher order electroweak processes. This is especially critical given that the extracted strange form factors appear to be rather small numerically $[1,2]$.

\section{$\gamma Z$ BOX CORRECTIONS TO PVES}

Beyond the Born approximation, the PV asymmetry receives corrections from higher order radiative effects, such as vertex corrections, wave function renormalization, vacuum polarization, and inelastic bremsstrahlung, which are well known and included in standard data analyses. Less well determined are radiative corrections arising from the interference of Born and two-boson exchange (TBE) diagrams, both electromagnetic $(\gamma \gamma)$ and electroweak $(\gamma Z)$. For purely electromagnetic scattering, the two-photon exchange corrections these have been shown $[5,6]$ to display strong angular dependence, which significantly affects extractions of the $G_{E}^{\gamma p} / G_{M}^{\gamma p}$ ratio by Rosenbluth separation [7].

The correction to the Born level PV asymmetry (2) can then be represented as $[8,9]$

$$
A_{\mathrm{PV}} \rightarrow(1+\delta) A_{\mathrm{PV}} \equiv\left(\frac{1+\delta_{\mathrm{Z}(\gamma \gamma)}+\delta_{\gamma(Z \gamma)}}{1+\delta_{\gamma(\gamma \gamma)}}\right) A_{\mathrm{PV}}
$$

Since the TBE corrections are typically only a few percent, the full correction $\delta$ can be written approximately as $\delta \approx \delta_{Z(\gamma \gamma)}+\delta_{\gamma(Z \gamma)}-\delta_{\gamma(\gamma \gamma)}$.

A comparison of the total TBE corrections $\delta$ with nucleon and $\Delta(1232)$ intermediate states is presented in Fig. 1 (left panel) as a function of $t$ for several fixed $\varepsilon$ values. At small $\varepsilon(\varepsilon \lesssim 0.6)$ the TBE correction at $|t| \lesssim 1 \mathrm{GeV}^{2}$ is dominated by the nucleon elastic contribution. At larger $\varepsilon$ the $\Delta$ plays an increasingly important role, and generally exceeds the nucleon piece at $\varepsilon \gtrsim 0.9$. However, for $\varepsilon \sim 1$ the calculation is sensitive to the high-energy behavior of the $\gamma N \Delta$ vertex, which because of the additional powers of momentum in the numerator, encounters problems with unitarity [10]. The calculation of the $\Delta$ contribution therefore becomes less reliable as $\varepsilon \sim 1$.

While the nucleon and $\Delta$ corrections are relatively small for $|t|$ between $\approx 0.01$ and $3 \mathrm{GeV}^{2}$, at very low $|t|$ there can be a sizable enhancement of the $\gamma Z$ contribution at extremely forward angles, $\varepsilon \rightarrow 1$, as pointed out by Gorchtein and Horowitz [11]. In this region the TPE contribution is suppressed, and the Born term is dominated by the proton weak charge, $Q_{W}^{p}$. Hence the $\Delta$ contribution would be enhanced by a factor 

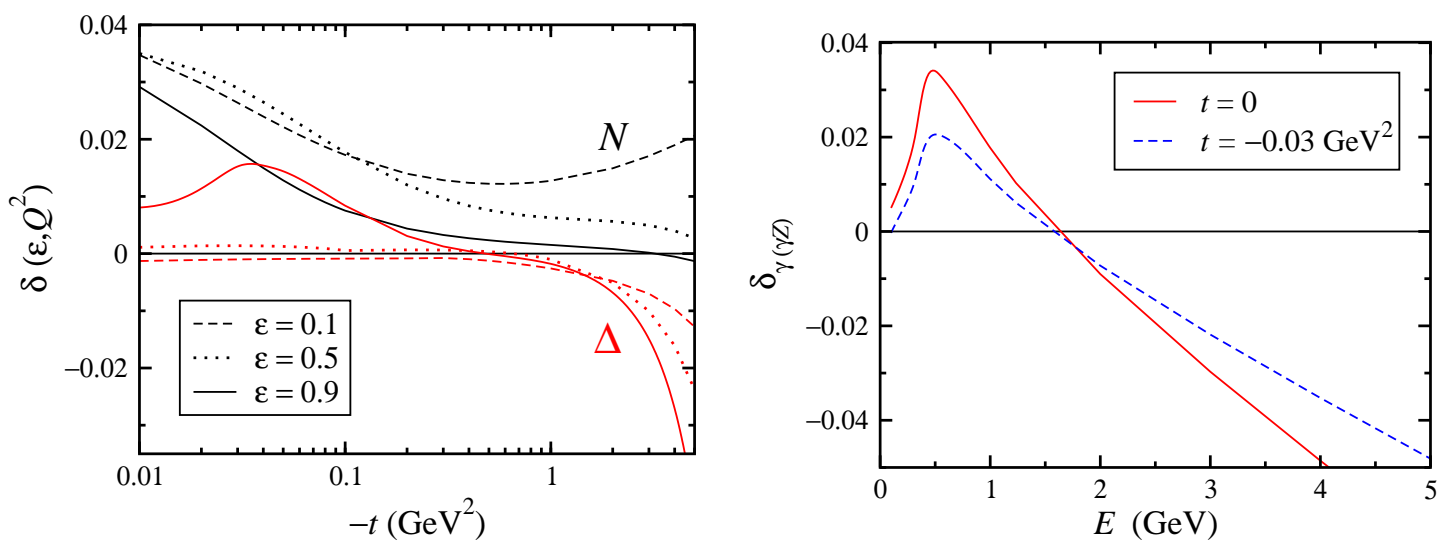

FIGURE 1. (Left) Total TBE corrections $\delta_{N}$ (upper three curves) and $\delta_{\Delta}$ (lower three curves) versus $-t$ for fixed $\varepsilon$ values, $\varepsilon=0.1$ (dashed), 0.5 (dotted) and 0.9 (solid). (Right) TBE correction $\delta_{\gamma(\gamma Z)}$ arising from nucleon and $\Delta$ intermediate states as a function of the incident electron energy $E$, for $t=0$ (solid) and $t=-0.03 \mathrm{GeV}^{2}$ (dashed) [9].

$\left(1+Q_{W}^{p}\right) / Q_{W}^{p} \approx 15$. In Fig. 1 the sum of the nucleon and $\Delta$ contributions to $\delta_{\gamma(\gamma Z)}$ is shown as a function of the incident electron energy $E$, for $t=0$ and for the Qweak [3] value $|t|=0.03 \mathrm{GeV}^{2}$. The $\Delta$ contribution rises linearly with energy up to $E \sim 0.5 \mathrm{GeV}$, where it reaches $\approx 2-3 \%$, after which it decreases.

The impact of these corrections on the strange form factors is difficult to gauge without performing a full reanalysis of the data, since in general different electroweak parameters and form factors are used in the various experiments. An estimate of the effects can be obtained by considering the dependence of the strange part of the asymmetry on input form factors. Differences of the order of $15 \%$ were found [8] between the empirical and monopole form factors for the HAPPEX kinematics [12, 13], and up to $30 \%$ for the PVA4 kinematics [14]. One should caution, however, that these values are indicative only, and a more detailed reanalysis of the strange form factor data including TBE effects is warranted.

\section{DISPERSIVE ANALYSIS OF $\gamma Z$ CORRECTIONS}

In the $t \rightarrow 0$ and low energy limits the PV asymmetry is related to the weak charge of the proton $Q_{W}^{p}$,

$$
A^{\mathrm{PV}} \rightarrow \frac{G_{F}}{4 \pi \alpha \sqrt{2}} t Q_{W}^{p}
$$

where the corrections are of $\mathscr{O}\left(t^{2}\right)$. Including electroweak radiative corrections, the proton weak charge is defined, at zero energy and momentum transfer, as [15]

$$
Q_{W}^{p}=\left(1+\Delta \rho+\Delta_{e}\right)\left(1-4 \sin ^{2} \theta_{W}(0)+\Delta_{e}^{\prime}\right)+\square_{W W}+\square_{Z Z}+\square_{\gamma Z},
$$

where $\sin ^{2} \theta_{W}(0)$ is the weak mixing angle at zero momentum, and the corrections $\Delta \rho$, $\Delta_{e}$ and $\Delta_{e}^{\prime}$ are given in [15] and references therein. The contributions $\square_{W W}$ and $\square_{Z Z}$ from 
the $W W$ and $Z Z$ box diagrams can be computed perturbatively, while the $\gamma Z$ interference correction $\square_{\gamma Z}$ in addition depends on physics at low momentum scales [16]. The current best theoretical estimate from Ref. [15] is $Q_{W}^{p}=0.0713(8)$.

The weak charge $Q_{W}^{p}$ extracted from the experimental asymmetry $A^{\mathrm{PV}}$ in Eq. (5) will differ from the zero-energy $Q_{W}^{p}$ in Eq. (6) by an amount $\square_{\gamma Z}(E)-\square_{\gamma Z}(0)$. In general the $\gamma Z$ box correction has contributions from the vector electron-axial vector hadron coupling of the $Z$ boson $\left(\square_{\gamma Z}^{A}\right)$ and from the axial vector electron-vector hadron coupling of the $Z\left(\square_{\gamma Z}^{V}\right), \square_{\gamma Z}(E)=\square_{\gamma Z}^{A}(E)+\square_{\gamma Z}^{V}(E)$. In their seminal early work, Marciano \& Sirlin [16] computed the $\square_{\gamma Z}^{A}(0)$ correction, which is dominant in atomic parityviolation experiments at very low electron energies, and has a weak energy dependence [17]. The vector-hadron $\gamma Z$ correction, $\square_{\gamma Z}^{V}(E)$, vanishes at zero energy, but aquires a strong energy dependence at nonzero $E[11,18]$, and is the focus of the discussion in the following.

Applying Cauchy's integral theorem, the real part of $\square_{\gamma Z}^{V}(E)$ can be obtained from its imaginary part using a standard dispersion relation,

$$
\mathfrak{R} e \square_{\gamma Z}^{V}(E)=\frac{1}{\pi} P \int_{-\infty}^{\infty} d E^{\prime} \frac{\mathfrak{I} m \square_{\gamma Z}^{V}\left(E^{\prime}\right)}{E^{\prime}-E},
$$

where $P$ is the principal value. The integration over negative energies corresponds to the crossed $\gamma Z$ box diagram, with the vector hadron correction even under $E^{\prime} \rightarrow-E^{\prime}$. Consequently $\Re e \square_{\gamma Z}^{V}(0)=0$, justifying the neglect of this term at atomic scale electron energies [16].

From the optical theorem, the imaginary part of the parity-violating $\gamma Z$ exchange amplitude can be written in terms of the vector interference structure functions,

$$
\mathfrak{I} m \square_{\gamma Z}^{V}(E)=\frac{\alpha}{\left(s-M^{2}\right)^{2}} \int_{W_{\pi}^{2}}^{s} d W^{2} \int_{0}^{Q_{\max }^{2}} \frac{d Q^{2}}{1+Q^{2} / M_{Z}^{2}}\left[F_{1}^{\gamma Z}+F_{2}^{\gamma Z} \frac{s\left(Q_{\max }^{2}-Q^{2}\right)}{Q^{2}\left(W^{2}-M^{2}+Q^{2}\right)}\right],
$$

where $s=M(M+2 E)$ is the total c.m. energy squared and $M_{Z}$ is the $Z$ boson mass. The structure functions $F_{1,2}^{\gamma Z}$ are functions of the exchanged boson virtuality, $Q^{2}$, and of the invariant mass $W$ of the exchanged boson and proton (or alternatively of the Bjorken variable $x=Q^{2} /\left(W^{2}-M^{2}+Q^{2}\right)$ ). The lower limit of the $W$ integration is given by the mass $W_{\pi}=M+m_{\pi}$ of the pion production threshold, and the upper limit of the $Q^{2}$ integration is given by $Q_{\max }^{2}=2 M E\left(1-W^{2} / s\right)$.

The definitions of the structure functions in Eq. (8) coincide with the standard definitions given by the PDG [19], in which at large $Q^{2}$ and $W$ the $F_{2}^{\gamma Z}$ structure function, for example, can be written (at leading order) in terms of parton distributions $q$ and $\bar{q}$ as $F_{2}^{\gamma Z}=x \sum_{q} 2 e_{q} g_{V}^{q}(q+\bar{q})$, with weak vector charges $g_{V}^{u}=1 / 2-(4 / 3) \sin ^{2} \theta_{W}$ and $g_{V}^{d}=-1 / 2+(2 / 3) \sin ^{2} \theta_{W}$.

Note that the expression (8) is a factor of 2 larger than that quoted in Ref. [11]. One can verify that Eq. (8) reproduces the known asymptotic limit for a point-like hadron [16], and also agrees with the independently calculated result for an elastic nucleon 
intermediate state $[8,9]$. The result (8) was also confirmed independently in Ref. [20]. The relation between the structure functions and the virtual photon total cross sections used in [11] also omits a factor $(1-x)$ relative to the usual definition, which leads to an overestimate of the contribution by $30-40 \%$ (and even more in the resonance region).

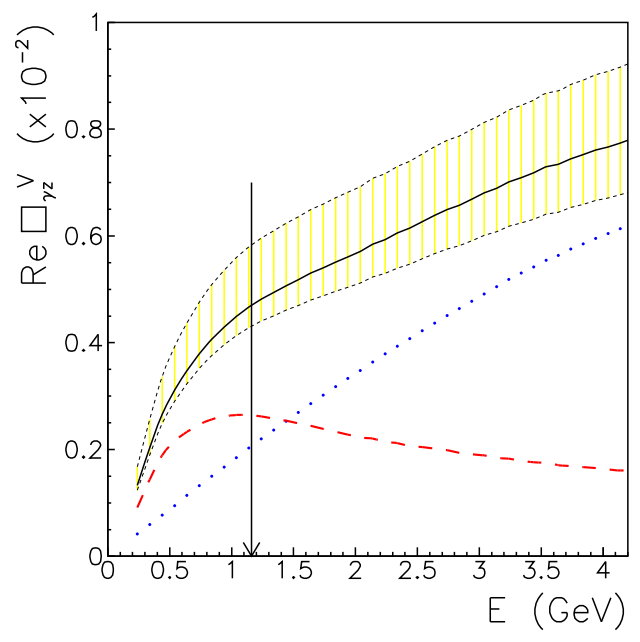

FIGURE 2. $\quad \gamma Z$ box correction $\square_{\gamma Z}^{V}(E)$ to $Q_{W}^{p}$ as a function of electron energy $E$, showing the resonant (dashed) and nonresonant (dotted) contributions, as well as the sum (solid) and the overall (asymmetric) uncertainty (shaded) [18]. The vertical arrow at $E=1.165 \mathrm{GeV}$ indicates the energy of the Qweak experiment.

Performing the dispersion integration in Eq. (7), the result for $\Re e \square_{\gamma Z}^{V}(E)$ is shown in Fig. 2 as a function of the incident electron energy $E$. Although the integration in principle involves an infinite range of $W^{2}$ and $Q^{2}$, in practice around $80 \%$ of the value of $\Re e \square_{\gamma Z}^{V}(E)$ at the energy relevant to Qweak comes from energies below $4 \mathrm{GeV}$, where the $Q^{2}$ range extends to $\sim 6 \mathrm{GeV}^{2}$, and $W$ to $\sim 3 \mathrm{GeV}$. This is fortunate as it is precisely in this region that a wealth of very accurate data exists from JLab [21, 22].

As seen in Fig. 2, the nonresonant contribution is small at low energies, but rises logarithmically with increasing $E$. The resonance contribution increases steeply to a maximum at $E \sim 1 \mathrm{GeV}$, before falling off like $1 / E$. The resonant and nonresonant contributions to $\Re e \square_{\gamma Z}^{V}(E)$ are 0.0026 and 0.0021 , respectively, at the energy relevant for the Qweak experiment, $E=1.165 \mathrm{GeV}$. One should note, however, that this separation is somewhat arbitrary, as only the physically meaningful, total cross section enters into our fit.

\section{DISCUSSION}

The combined correction from the $\gamma Z$ box contribution $\Re e \square_{\gamma Z}^{V}$ to $Q_{W}^{p}$ at the Qweak energy is then $0.0047_{-0.0004}^{+0.0011}$, or $6.6_{-0.6}^{+1.5} \%$ of the Standard Model value $0.0713(8)$ for $Q_{W}^{p}$, with the error band obtained from the uncertainty in the fit parameters using a variational method [18]. In comparison, the correction found in Ref. [11] was $\approx$ 
0.003. The major difference between these value arises from the additional factor 2 in Eq. (8), which has been verified independently [20], as well as our use of more recent electroproduction data. The correction is important for the interpretation of the Qweak experiment, given its projected uncertainty of \pm 0.003 [3]. It is also critical to the physical interpretation of the experiment which is expected to constrain possible sources of parity violation from beyond the Standard Model at a mass scale of $\gtrsim 2 \mathrm{TeV}$ [4]. The main uncertainty in the calculation, namely the $\gamma Z$ interference structure functions, can be further reduced in the future by incorporating new inclusive parity-violating data in the resonance and deep inelastic scattering region which are planned at Jefferson Lab [23].

\section{ACKNOWLEDGMENTS}

I would like to thank P. B. Blunden, A. Sibirtsev, A. W. Thomas and the late J. A. Tjon for collaboration on the topics presented in this talk. This work was supported by the DOE contract No. DE-AC05-06OR23177, under which Jefferson Science Associates, LLC operates Jefferson Lab. I gratefully acknowledge support from the ARC International Linkage Award LX0776452 to attend this workshop.

\section{REFERENCES}

1. R. D. Young, J. Roche, R. D. Carlini and A. W. Thomas, Phys. Rev. Lett. 97, 102002 (2006).

2. J. Liu, R. D. McKeown and M. J. Ramsey-Musolf, Phys. Rev. C 76, 025202 (2007).

3. Jefferson Lab experiment E-08-016 (Qweak), R. D. Carlini et al. spokespersons.

4. R. D. Young, R. D. Carlini, A. W. Thomas and J. Roche, Phys. Rev. Lett. 99, 122003 (2007).

5. P. G. Blunden, W. Melnitchouk and J. A. Tjon, Phys. Rev. Lett. 91, 142304 (2003).

6. P. G. Blunden, W. Melnitchouk and J. A. Tjon, Phys. Rev. C 72, 034612 (2005).

7. J. Arrington, W. Melnitchouk and J. A. Tjon, Phys. Rev. C 76, 035205 (2007).

8. J. A. Tjon and W. Melnitchouk, Phys. Rev. Lett. 100, 082003 (2008).

9. J. A. Tjon, P. G. Blunden and W. Melnitchouk, Phys. Rev. C 79, 055201 (2009).

10. P. G. Blunden et al., in preparation.

11. M. Gorchtein and C. J. Horowitz, Phys. Rev. Lett. 102, 091806 (2009).

12. K. A. Aniol et al., Phys. Rev. C 69, 065501 (2004).

13. A. Acha et al., Phys. Rev. Lett. 98, 032301 (2007).

14. F. E. Maas et al., Phys. Rev. Lett. 94, 152001 (2005)

15. J. Erler, A. Kurylov and M. J. Ramsey-Musolf, Phys. Rev. D 68, 016006 (2003); J. Erler and M. J. Ramsey-Musolf, Phys. Rev. D 72, 073003 (2005).

16. W. J. Marciano and A. Sirlin, Phys. Rev. D 27, 552 (1983); ibid. 29, 75 (1984) [Erratum ibid. D 31, $213(1985)]$.

17. P. G. Blunden, W. Melnitchouk and A. W. Thomas, in preparation.

18. A. Sibirtsev, P. G. Blunden, W. Melnitchouk and A. W. Thomas, Phys. Rev. D 82, 013011 (2010).

19. C. Amsler et al., Phys. Lett. B 667, 1 (2008).

20. B. C. Rislow and C. E. Carlson, arXiv:1011.2397 [hep-ph].

21. M. Osipenko et al., Phys. Rev. D 67, 092001 (2003).

22. I. Niculescu et al., Phys. Rev. Lett. 85, 1186 (2000); Y. Liang et al., arXiv:nucl-ex/0410027; S. P. Malace et al., Phys. Rev. C 80, 035207 (2009).

23. Jefferson Lab experiments E-05-007, R. Michaels, P. Reimer and X. Zheng spokespersons, and E1207-102, K. Paschke, P. Reimer and X. Zheng spokespersons. 